\title{
Digital Twin: An Oracle for Efficient Crowdsourcing of Research \& Technology Development through Blockchain
}

Jens Ducrée

School of Physical Sciences, Dublin City University, Glasnevin, Dublin 9, Ireland, email: jens.ducree@dcu.ie

\section{Abstract}

Since its inception in the late 2000 s, blockchain has emerged as a powerful tool for creating trust without intermediaries to incentivize global communities for working for a common goal, such as the improvement of its very ecosystem, its applications and community adoption. While first blockchains were mainly devised for confirming transactions of their innate cryptocurrencies like Bitcoin, smartcontract blockchains like Ethereum can interface with the real-world through so-called "oracles", which feed trustful off-chain information. This paper introduces digital twins of physical objects and processes as computational oracles to effectively unleash the tremendous opportunity offered by blockchain to the realm of fundamental science, research and technology development (RTD). The crowdsourcing concept is illustrated with the example of centrifugal flow control in microfluidic "Labon-a-Disc" (LoaD) systems.

Keywords

Digital twin, centrifugal microfluidics, Lab-on-a-Disc, crowdsourcing, blockchain, decentralization, oracle, consensus, non-fungible token, NFT, decentralized science, DeSci

\section{Introduction}

The distributed ledger technology blockchain is on the verge of disrupting the way economies and societies are organized. Since the launch of the world's first (widely adopted) cryptocurrency "Bitcoin" by a still mysterious pseudonym persona "Satoshi Nakamoto", a moniker for a still unidentified individual or group, in 2008/2009 [1], underlying blockchain technologies have largely diversified and grown at an unprecedented pace that even dwarfs the massive proliferation of the internet starting in the 1990s. Bitcoin's original designation was a peer-to-peer payment network that is detached from monetary policies of governments, the printing of fiat currencies by their central banks.

By now, Bitcoin still leads crypto market capitalization on the order of 1 trillion US\$, is increasingly adopted as store of value by professional and retail investors, and even accepted or seriously considered as a legal tender (based on $2^{\text {nd }}$-layer fast, low-fee payment solutions) in a first cohort countries like El Salvador, Paraguay, Panama, Venezuela and Ukraine, usually as an alternative to the US dollar as their present, de facto store-of-value currency [2,3]. While different forms of crypto-bans and regulations are proposed or have been imposed in several economically very significant countries [4-6], and high price volatility somehow persists, Bitcoin and leading altcoins has so far impressively recovered from many of such setbacks.

Another hallmark underpinning modern blockchain technologies was the introduction of smart contracts [7, 8] with the launch of the Ethereum blockchain in 2015 [9]. By staking, rewarding and slashing of cryptographically secured "coins", the token economy ("tokenomics") enabled by such 
"programmable money" incentivizes "good behavior" to endow solid trust across a highly decentralized community of diverse, and potentially anonymous participants and governance bodies.

As compelling evidence of the huge trust generated without a central coordinator and regulator, the total value locked (TVL) in the cryptocurrencies entertained on the currently dominant blockchains amounts to about 2 trillion US\$ at the time of writing.

In addition to Ethereum, various smart-contract blockchains have crystalized in terms of their highestranking market capitalization (status: October 2021) [10-15]. While it is virtually impossible to predict the winner(s) amongst the present and possible new emerging technologies, one of the overarching objectives remains interoperability in a future internet of blockchains. Another trend is that only Bitcoin sticks to its electricity-consuming "Proof-of-Work" [16], with an increasing number of mining shifting towards using renewables, most other blockchains have or will implement alternative strategies, including (delegated) "Proof-of-Stake" [17], "Proof-of-Authority" or "Proof-of-History", for securing the integrity of their chains.

With its cornerstones in computer science, cryptography and finance, state-of-the-art blockchain technology is distinctively multi-facetted; its further sophistication and application development involve a wide repertoire of fields encompassing economics, game theory, banking, risk management, data science, education, law, administration, political science, psychology, ethics, arts and social sciences.

Blockchain technologies have proven to bestow trust in virtual assets like cryptocurrencies, smart contracts and non-fungible tokens (NFTs), e.g., representing provenance and ownership of unique digital or physical artefacts, artwork and intellectual property (IP), that are stored and managed without the need for intermediaries within its own ecosystem.

So-called "oracles" provide a real-world interface for blockchains [18-22]; they provide smart contracts with external "off-chain" information, such as the status of a financial transaction, market prices for stocks, commodity or currency prices, weather data, election outcomes, sports results, or coordinated time. Especially in the context of rapidly evolving "Internet of Things" (IoT) applications, also tamper-proof solutions for supplying credible physical data, such as local temperature, humidity, wind speed, atmospheric pressure, or live traffic data that are, for example, essential for a green economy, have been incentivized by blockchain-based tokenomics [23, 24].

Oracles are essential for unleashing the full potential of blockchain beyond its own digital habitat. There are various types of oracles, which can be categorized according to the source, i.e., software and hardware, direction, i.e., inbound and outbound, and anchoring, i.e., centralized, decentralized, or human-based [21]. For code-based decision making, off-chain oracles typically form the weakest link in the trust chain, even leading to possibly fatal fallout; therefore, and also in the framework of this work, creating trust in oracles is absolutely mission critical.

Blockchain has also been very successful to organize crowdsourcing and governance for the development of its own environment and application space, for instance, by decentralized autonomous organizations (DAOs) [25-30], which, after some serious early setbacks [31, 32], have gone through a steep learning curve in the meantime.

This work aims to adopt the lessons learnt from highly complex crowdsourced development and community governance in various blockchains to science, research and technology development (RTD), where project outcomes can often not be represented by a single value that can be properly 
validated in decentralized manner, the development of concepts to endow trust as a key requisite for management by blockchain remains a major challenge.

This paper outlines how digital twin technologies [33-35] assume, in a first step, a paramount role in leveraging the enormous benefits of blockchain-enabled crowdsourcing for science and RTD. There are two chief ingredients for success: The advancement of the usually complex and interdisciplinary modelling underlying the digital twin, including its foundational mathematical equations, simulation methods and techno-economical boundary conditions, e.g., imposed by manufacturing or economical restrictions. Documented in a "whitebook", the model is then progressively refined and independently validated by the community. In a second step, the goals of the research project that are expressed by quantitative key performance indicators (qKPIs). Then, literally anybody can optimize a set of design parameters, and thus contribute to the, possibly iterative, progress towards the well-defined objectives. In a decentralized mechanism referred to in the following as "computational oracle", the community then verifies and ranks the outcomes predicated on the jointly accepted digital twin. The project information, participation and (read-only) access may be open to the public, or accessrestricted.

This paper first expands the main concept of using the digital twin as a computational oracle to verify, and thus fairly reward crowdsourced contributions to science and RTD projects. Then the modelling enabling the digital twin is demonstrated along the specific instance of centrifugal microfluidic flow control. Next, the chief elements for entertaining crowdsourcing of validation, refinement and performance optimization this "Lab-on-a-Disc" (LoaD) technology are explained. Finally, its application cases and possible extensions are pointed out before summarizing and articulating objectives for future work.

\section{Digital Twin as Computational Oracle}

This paper suggests digital twins as a special type of virtual oracle that offer the missing link for harnessing the advantages of blockchain-based crowdsourcing for science \& RTD, thus massively improving projects from idea generation, efficient development and optimization, credible validation and their widespread adoption. The keystone of the underlying concept (Figure 1) revolves about the computational oracle enabled by the digital twin.

\section{Central Idea}

An increasing number of real-world objects and processes can be modelled by a set of mathematical relations. Consequently, with proper knowledge of (ideally) all mathematical equations and their spatio-temporal boundary conditions, and equipped with sufficient computing power, digital twins can accurately describe the characteristics of a fully-defined system in the physico-chemical and biological domains, and algorithmically optimize the system according to a set of well-communicated performance metrics. 


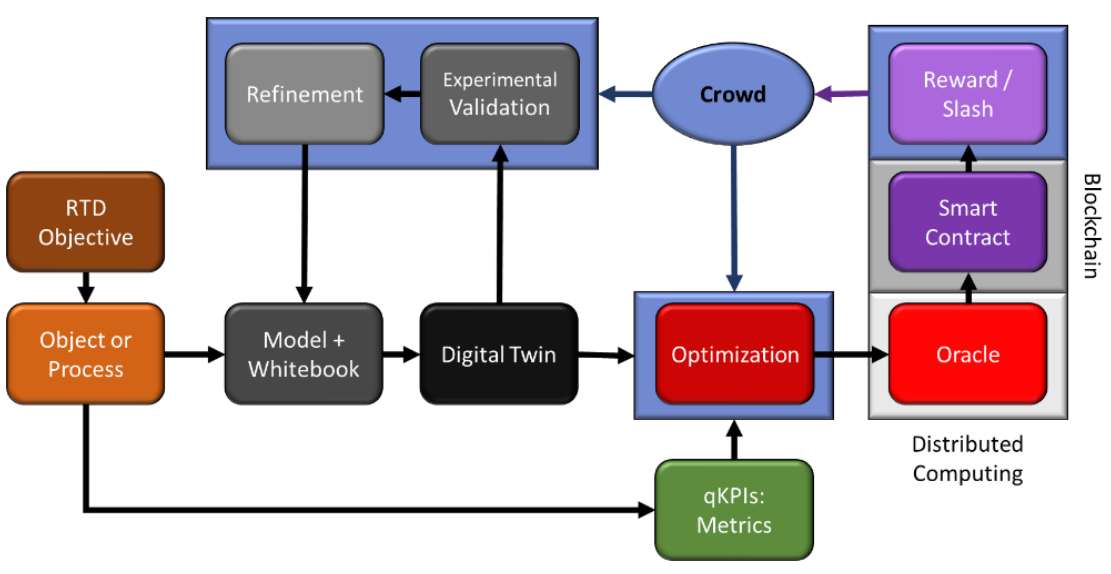

Figure 1 Computational oracle. The initiators of an RTD project, e.g., individuals, interest groups, investors, or societies, formulate its objectives. The target properties of the object or process is well defined by a collection of quantitative, and hence unambiguously verifiable metrics. Within a whitebook, the mathematical framework of a model is drafted, and amended by practical boundary conditions and the simulation methods. In a feedback loop, the crowd experimentally validates and refines this descriptor, so it best captures the real-world characteristics of the object or process. The optimization of the parameters towards the KPIs that were set out by the project initiators is also carried out decentrally by the crowd; as a decisive element of the concept, the evaluation of delivered parameters is implemented by a computational oracle simulating the digital twin within a decentralized network, or virtual machine; "good behavior" of all players is incentivized by demanding staked cryptoassets, possibly for a given locking period. Based on the outcomes, contributors are rewarded, or lose (part of) their stake, according to the conditions transparently encoded in the blockchain-engraved smart contract.

In practice, most mathematical models are, and may even never reach perfection; yet, resulting gaps might be adequately bridged by approximate formulas or fits to statistically sound empirical data. It turns out that virtual representations by digital twins are more suitable for fields like engineering and physics, while other disciplines, e.g., the life or social sciences, usually lack enough (quantitative or even quantitative) knowledge of the state of systems and the full gamut of formulas describing some important interactions.

However, with the exponential evolution and digitalization of science \& RTD, it can be well anticipated that the complexity of systems that can be meaningfully represented by digital twin models will, prospectively, greatly expand, thus markedly broadening the application space of the approach presented here.

\section{Phased Approach}

\section{Model Development}

There are two pivotal steps to establish the digital twin of a given system as a computational oracle for blockchain-based science \& RTD in a decentralized environment without intermediaries. First, the multi-domain modelling underpinning the digital twin is set up, possibly initiated by a group of experts. The mathematical descriptor is then iteratively sophisticated and validated by comparison between simulation and experiment, for its (sufficiently) accurate representation of the real world within reasonable ranges of its input parameters. Similar to introducing upgrades of blockchain code, refining the model might be organized by community-voted "improvement proposals", such as BIPs for Bitcoin [36], or EIPs for Ethereum [37].

\section{Performance Optimization}

Second, the resulting digital twin is employed to find the optimum parameter set, while factoring in reasonable restrictions, for the target application of the science \& RTD project according to quantitative key performance indicators (KPIs) that have been properly specified with numerical methods to be employed in the public or access-restricted whitebook issued with the project. In a 
feedback loop, potentially critical deviations between simulated and experimental outcomes need to be thoroughly investigated to iteratively hone the underlying model and computational methods. Yet, a well-tested model coming out from the first phase should widely eliminate such discrepancies.

\section{Consensus, Trust and Governance}

Both of the above steps constitute paramount components for unleashing the enhanced efficiency, credibility and adoption of research projects via the blockchain-based crowdsourcing paradigm. Yet, this novel approach requires good mechanisms for establishing trust, for finding consensus, and for implementing governance within its smart-contract encoded framework of potentially anonymous, globally distributed participants.

Blockchain already provides essential trust without the need for centralized bodies or middlemen. Inputs to the distributed ledger require the staking, e.g., of digital assets or computing resources; in a "betting game", such "skin-in-the-game" is rewarded, or slashed, by issuing system-innate cryptocurrencies, depending on the credibility, quality or speeds of delivered inputs to the peer-topeer ("P2P") network. The gravity of such stakes might be modulated by a (minimum) locking period. The value of the transacted crypto-assets is determined in a free, supply-and-demand market on exchanges, and their "tokenomics", including pre-launch allocations, e.g., to the team and investors, and by inflationary policies, is hardcoded in the blockchain. Such cryptographical incentivization has proven to be consistently functional for securing "unspent transaction output" (UTXO) at the very base layer of crypto-payments, governance decisions, e.g., on upgrades of the blockchain program, for creating prediction markets [38], and for feeding off-chain data to algorithmically decision-making oracles $[19,20,23]$.

Tasks may be posted, for instance, via bounties for improving the blockchain and its governance and consensus mechanisms, for experimental and virtual validation, for spotting bugs or proposing fixes; filing of intellectual ownership, e.g., for code modules by so-called non-fungible tokens (NFTs) [39-41], may non-repudiatively registered on the time-stamped and tamper-proof distributed ledger blockchain, and possibly amended by stake-based governance and arbitration through the interplay of community voting and councils [42-50]. In a more academic context, reputations systems [51] that are already familiar in the scientific community, such as the notorious h-index [52], may complement cryptocurrencies for staking and incentivization.

There are different ways how to implement the voting, and to tackle the potential issue of (mostly unwanted) monopolization in a decentralized community. Bitcoin employs an "economic" voting where miners enclose their ballot in the blocks which they get to produce over a given time period. A simple one-person-one-vote principle faces the issue of verifying personal identities of anonymous wallets addresses to avoid multiple votes. Several proposals for verifying identities may be adopted, for instance, following online identify verification on the analogy of know-your-customer ("KYC") in traditional banking ("TradFi").

There are also concepts how so-called zero-knowledge proofs ("ZKP") can implement identity verification in decentralized systems while protecting user privacy. While serious concerns have been pointed out on the feasibility of such techniques in democratic elections [53], community voting for finding consensus on matters on blockchain ecosystems and various oracles have been successfully implemented in blockchain. Alternative procedures, such as quadratic voting, which tapers stakebased voting power towards increasing assets [54-56], have been elaborated. 


\section{Computational Oracle}

A reward system is needed for crowdsourcing performance-optimized parameter sets, which can typically only be obtained from highly resource-demanding, and thus costly computations. Results originating from a participants need to be evaluated and ranked by community consensus.

Similar to PoW blockchains like Bitcoin and its cryptographic puzzles, necessary calculations are asymmetric, in a sense that arriving at optimized solutions involves substantially larger computational power (by the miner) than their confirmation by distributed nodes that can be installed and run, in principle, by anyone meeting the preset specifications. To protect the integrity of the verification, it is required that a sufficient number of independent validators execute the same model and numerical procedures according to (the latest, community-approved version of) the publicly documented whitebook; ideally, also the computing hardware should comply with certain standards, and the conformity of the chips may even be certified, e.g., along Trusted Platform Module (TPM) schemes [57].

Based on the consensus mechanism, e.g., simple majority, the rewards are transferred, according to a pre-defined allocation algorithm saved on the blockchain, to the contributing member(s) who came up with the improved parameter set(s); these pay-outs thus compensate for the investment in computing equipment and energy by the members of the crowd who optimized, validated, or staked in the process of consensus finding.

\section{Example: Lab-on-a-Disc}

Previous work has outlined how to leverage the benefits of blockchain-based community approaches for science \& RTD [58-63] in a more general context. This paper exemplifies the digital twin concept for the case of centrifugal microfluidic flow control, which has been experimentally demonstrated and elaborated in much more technical detail elsewhere [64-69].

\section{Fluidic Modelling}

The motion and reshaping of liquids and gases in response to pressure fields are commonly modelled by continuum mechanics. In microfluidic systems, liquids and gases are typically confined to mesoscale structures on mid micro- to lower millimeter range made of polymers. By virtue of the large surfaceto-volume ratios in the wake of miniaturization, laminar conditions prevail to greatly simplify fluid dynamics. Amongst the numerous microfluidic technologies developed since the early 1990s, centrifugal microfluidic systems have proven to provide comprehensive sample-to-answer automation of complex analytical test protocols on biofluids, e.g., taken from patients, agrifood, infrastructures, industry, or the environment.

Most such "Lab-on-a-Disc" (LoaD) systems operate in batch mode, in a sense that Laboratory Unit Operations (LUOs), such as plasma extraction from whole blood or volume metering and mixing with reagents, are carried out while the liquids are retained by downstream valves. The flow control technologies considered in this showcase are merely actuated by the (angular) speed of rotation $\omega=$ $2 \pi \cdot v$, more specifically by "high-pass" burst valves that open upon exceeding a critical frequency, i.e., $\omega>\Omega$.

Applications of microfluidic systems often involve the concurrent management of several liquids and reagents in multi-step liquid handling protocols at the backbone of bioassay automation. This requirement poses a particular challenge to centrifugal systems where all liquids residing on the given rotor are exposed to the same spin rate $\omega$. Fluidic multiplexing by the high-pass valves thus involves 
staggering of discrete valving frequencies that are ordered according to their intended release sequence.

The fluidic mechanism underlying the valving concept establishes the burst rate $\Omega$ as a function of its experimental input parameters, which are collectively referred to as $\left\{\gamma_{k}\right\}$. Decisively, to reflect realworld circumstances, this digital twin for the LoaD also accounts for statistical variations $\left\{\Delta \gamma_{k}\right\}$ in $\left\{\gamma_{k}\right\}$, such as unavoidable dimensional tolerances in manufacturing of fluidic structures, and in the definition of liquid volumes. Overall, the set of discrete valving frequencies $\left\{\Omega_{i}\right\}$ for each simultaneous release step $i$ smear outs into finite bands $\left\{\Omega_{i} \pm M \cdot \Delta \Omega_{i}\right\}$ with standard deviations $\left\{\Delta \Omega_{i}\right\}$; reliability of multiplexed valving thus hinges upon avoiding mutual overlap of these bands with the factor $M=$ $1,2,3, \ldots$ determining the confidence of proper functioning of stage $i$ according to the well-known 6895-99.7 percent rule.

\section{Centrifugo-Pneumatic Dissolvable-Film Siphon Valve}

In a specific example, we consider the transport of a discrete liquid volume $U$ and density $\varrho$ confined between its inner- and outermost radial extensions $\check{r}$ and $\hat{r}$, respectively, residing on a disc of a $12-\mathrm{cm}$ diameter of a conventional optical data storage medium like CD, DVD or Blu-ray. When spinning at $\omega$ in a centrifugo-pneumatic siphon valving structure $\Gamma$ (Figure 2 ) that is placed at the radial position $R$, the volume $U$ experiences a centrifugally induced pressure head

$$
p_{\omega}(U, \varrho, R, \Gamma, z, \omega)=\varrho \cdot \bar{r}(U, R, \Gamma, z) \Delta r(U, R, \Gamma, z) \cdot \omega^{2}
$$

with the mean radial position $\bar{r}=0.5 \cdot(\check{r}+\hat{r})$ and the liquid level difference $\Delta r=\hat{r}-\check{r}$ also depending on the axial position of the (forward) meniscus $z$, with $\check{r}=\operatorname{Min}\left[r_{0}(\omega), r(\omega)\right]>R$, and $\hat{r}=$ $R+h$ for $\Gamma$ as depicted in Figure 2 .

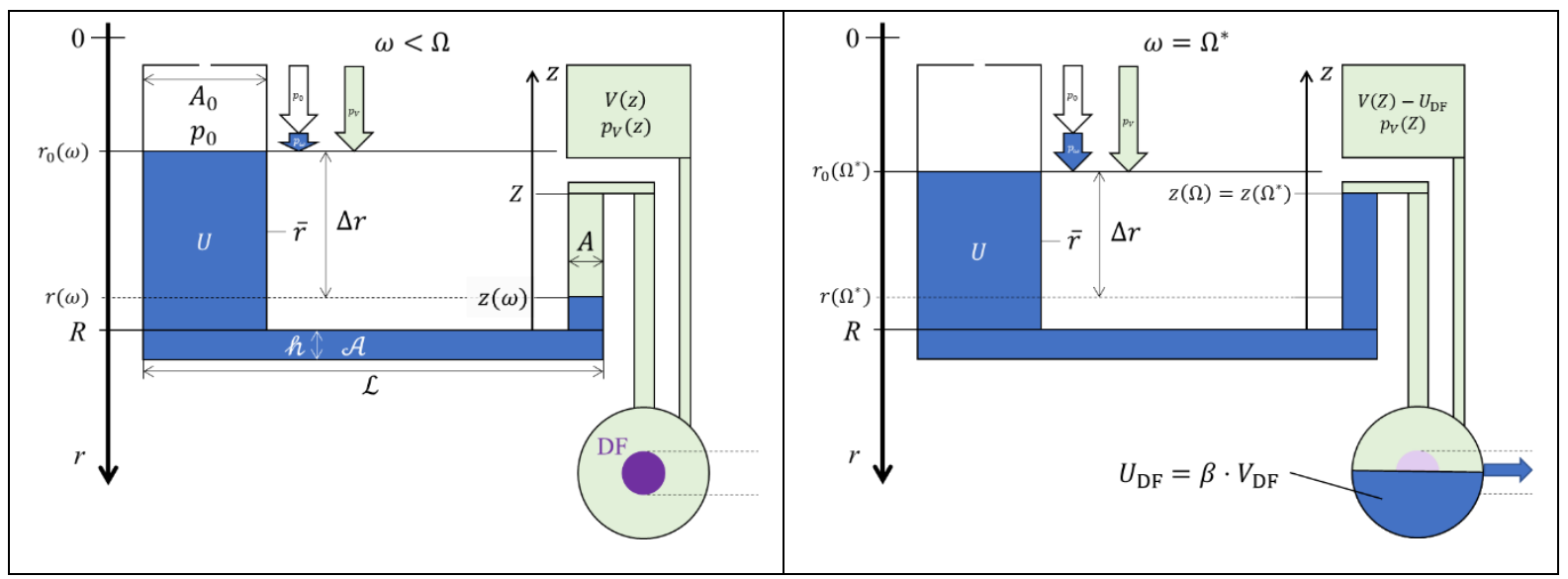

Figure 2 Centrifugo-pneumatic dissolvable-film siphon valve. The structure $\Gamma$ (linearized display with cuboidal segments, not to scale) is composed of an inlet that is open to atmosphere, an inbound siphon section, and an interconnecting isoradial channel of cross sections $A_{0}, \mathcal{A}$ and $A$ with respect to the axial direction $z$, respectively. The valve is loaded with a liquid volume $U$, so that the compression volume $V$ is isolated at ambient pressure $p_{0}$ once the inbound channel of total volume $\mathcal{A} \cdot \mathcal{L}$ and radial height $h$ is completely filled at the level $z=0$. (Left) At a spin rate $\omega<\Omega$, a centrifugo-pneumatic equilibrium (4) establishes between $p_{\omega}(1)$ and $p_{0}$ on the left, and $p_{V}(3)$ on the right. This hydrostatically balanced state is delineated by radial meniscus positions in the inlet reservoir $r_{0}(\omega)$, and the inbound segment $r(\omega)$, i.e., $0<z(\omega)=R-$ $r(\omega)<Z$. (Right) The critical spin rate $\Omega(5)$ is defined by $z(\Omega)=Z$, i.e., when the forward meniscus $r$ has reached the top of the inbound siphon section, just before overflowing. The valve opens at $\Omega^{*}>\Omega$, i.e., when a minimum liquid volume $U_{\mathrm{DF}}=\beta \cdot V_{\mathrm{DF}}$ has entered the DF chamber of dead volume $V_{\mathrm{DF}}$, with the coefficient $\beta$ representing the fractional filling required to wet the (centrally placed) DF.

Using the continuity of the (incompressible) liquid volume

$$
U=2 \pi \int_{\check{r}}^{\hat{r}} r \cdot \varrho(r) d r=\text { const. }
$$


over its liquid density distribution $\varrho(r)$ across the disc, the radial product $\langle\bar{r} \Delta r\rangle(\check{r}, \hat{r})=$ $\langle\bar{r} \Delta r\rangle(U, R, \Gamma, z)$ appearing in (1) can formally be written as a function of $U, R, \Gamma$ and $z$.

In centrifugo-pneumatic valves, $p_{\omega}(1)$ drives the mean position $\bar{r}$ of the liquid distribution radially outwards until it is balanced by the counterpressure

$$
p_{V}\left(p_{0}, \Gamma, z\right)=p_{0} \cdot \frac{V_{0}}{V(\Gamma, z)}
$$

resulting from the compression of an enclosed gas pocket from an initial volume $V_{0}=V(z=0)$ at (ambient) pressure $p_{0}$ to $V(z>0)$ according to Boyle's law.

\section{Retention Frequency}

For an open inlet reservoir, as displayed in Figure 2, the hydrostatic equilibrium is expressed by

$$
p_{\omega}+p_{0}=p_{V}
$$

which interrelates the variables $p_{0}, U, \varrho, R, \Gamma, z$ and $\omega$. From (4), a burst frequency

$$
\Omega\left(p_{0}, U, \varrho, R, \Gamma, Z\right)=\sqrt{\frac{p_{V}-p_{0}}{\varrho \cdot \bar{r} \Delta r}}
$$

can be inferred for halting the liquid at a given critical axial position upstream of $z=Z$. While the parameters $U$ and $\varrho$ are usually prescribed by the assay protocol, and $p_{0}$ by the ambient pressure depending on local weather and altitude at the time of executing the test, and $R$ to some extent by the position of the associated LUO in the assay protocol, $\Omega(5)$ can mainly be tuned by the geometrical parameters $\Gamma$ and $Z$.

For opening the CP-DF siphon valve inFigure 2, (right), the spin rate is (slightly) elevated to $\Omega^{*}>\Omega$ for displacing a liquid volume $U_{\mathrm{DF}}=\beta \cdot V_{\mathrm{DF}}$ in the outer chamber of dead volume $V_{\mathrm{DF}}$ accommodating the DF. For the shown, central placement of the DF, we obtain $\beta=0.5$. Formally, $\Omega^{*}$ follows the same formula as $\Omega(5)$, after replacing $U$ by $U-U_{\mathrm{DF}}$, and $V(Z)$ by $V(Z)-U_{\mathrm{DF}}$ for calculating the radial product $\bar{r} \Delta r$ in (1) and $p_{V}$ (3), respectively. For the sake of simplicity in this brief outline, we do not distinguish between $\Omega$ and the slightly elevated $\Omega^{*}$.

\section{Band Width}

In general, the standard deviation $\Delta \Omega$ of the burst frequencies $\Omega$ depends on the statistical spreads $\left\{\Delta \gamma_{k}\right\}$ of its input parameters $\left\{\gamma_{k}\right\}$; often, these are mainly associated with the (possibly distinct) vertical and lateral machining tolerances $\Delta \Gamma$, and the volume definition $\Delta U$. In the digital twin, virtual testing incorporating $\Delta \Gamma$ for calculating actual values $\widetilde{\Omega}$ may directly be derived from (a sufficient number of) Monte-Carlo simulation runs. However, for algorithmic design optimization, e.g., to minimize $\Delta \Omega$, it is more useful to utilize Gaussian error propagation

$$
\Delta \Omega\left(\gamma_{k}, \partial \Omega / \partial \gamma_{k}, \Delta \gamma_{k}\right)=\sqrt{\sum_{k}\left(\frac{\partial \Omega}{\partial \gamma_{k}}\right)^{2} \cdot \Delta \gamma_{k}^{2}}
$$

which, strictly speaking, only holds for independent variables $\left\{\gamma_{k}\right\}$ and small $\left\{\Delta \gamma_{k}\right\}$. Note that while the formal derivation of the opening frequency $\Omega$ (5), and its standard deviation $\Delta \Omega$ (6), looks straight forward, finding solutions reflecting the complex dependency of the radial product $\bar{r} \Delta r$ (1) on $\Gamma$ likely involves extensive numerical calculations. 


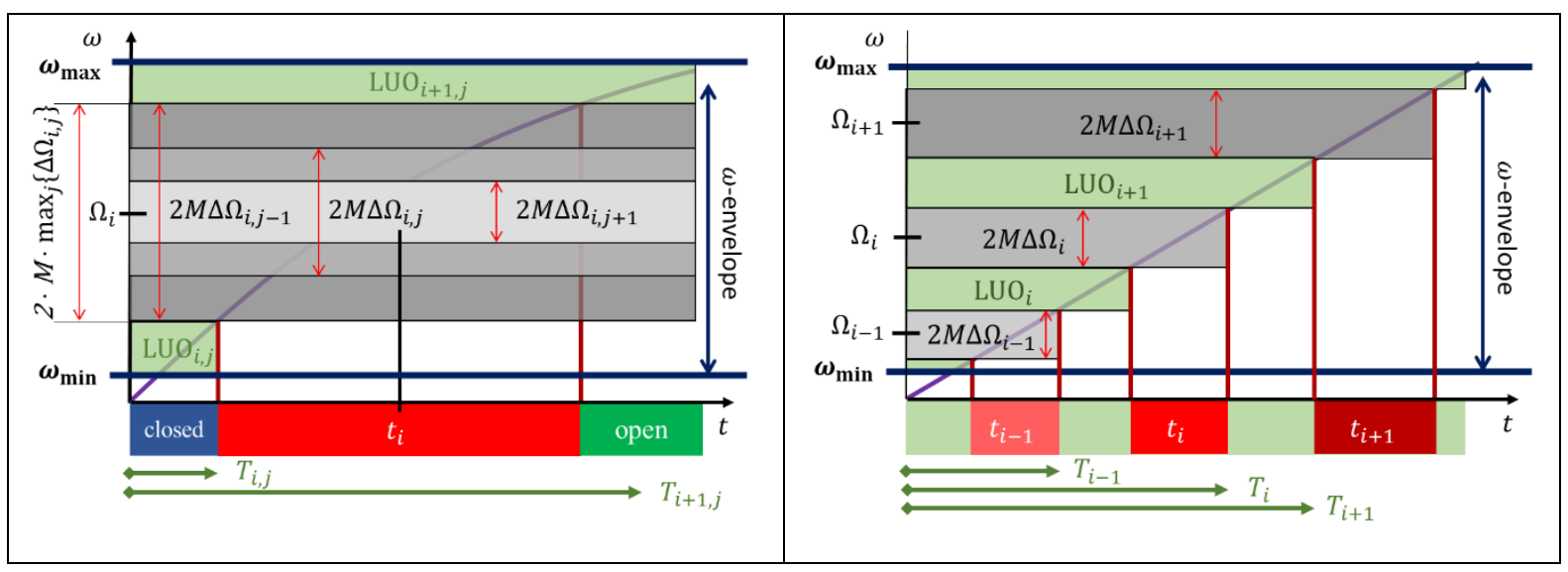

Figure 3 Distribution of valving structures $\left\{\Gamma_{i}\right\}$ possessing bands $\left\{\Omega_{i} \pm M \cdot \Delta \Omega_{i}\right\}$ in frequency space. The LoaD system implements multiplexed flow control by high-pass valves, e.g., for the centrifugo-pneumatic dissolvable-film siphon schemes $\Gamma$ schematized in Figure 2. (Left) Simultaneous actuation around $t=t_{i}$ of three valves $\left\{\Gamma_{i, j}\right\}$ tuned via (5) for the same nominal burst rate $\Omega_{i}=\Omega_{i, j-1}=\Omega_{i, j}=\Omega_{i, j+1}$, but displaying different standard deviations, here $\Delta \Omega_{i, j-1}<\Delta \Omega_{i, j}<$ $\Delta \Omega_{i, j+1}$. For robust flow control, the rotational frequency $\omega$ needs to cross the "forbidden zone" extending between $\min _{j}\left\{\Omega_{i, j}-\Delta \Omega_{i, j}\right\}$ and $\max _{j}\left\{\Omega_{i, j}+\Delta \Omega_{i, j}\right\}$. (Right) Sequential actuation for bands $\left\{\Omega_{i} \pm M \cdot \Delta \Omega_{i}\right\}$ at $t_{i-1}<t_{i}<t_{i+1}$ that are separated green spaces that are used for executing the upstream LUOs during the periods of length $T_{i}$. These bands of widths $\left\{2 \cdot M \cdot \Delta \Omega_{i}\right\}$ need to be staggered while avoiding mutual overlap. Similar to the available physical surface between $R_{\text {min }}$ and $R_{\max }$ restricting number of structures $\Gamma$ that can be integrated on a LoaD device, also the maximum degree of multiplexing that can be implemented at the required operational reliability, as quantified by the factor $M$, within the practically allowed frequency envelope $\omega_{\min } \leq \omega \leq \omega_{\max }$, is limited.

Figure 3 shows the distribution of spin rate bands $\left\{\Omega_{i} \pm M \cdot \Delta \Omega_{i}\right\}$ that are deliberately positioned and separated in frequency space to allow simultaneous (left) and sequential (right) release of corotating liquid volumes $U_{i}$ of the burst valves $\Gamma_{i}$. The LUOs $i$ are carried out over a time $T_{i}$ in the green-regions of the respective frequency domains so that $\Omega_{i}+M \cdot \Delta \Omega_{i}<\omega<\Omega_{i+1}+M \cdot \Delta \Omega_{i+1}$. The band widths should thus be reduced to maximize the degree of multiplexing that can be squeezed into the practically available spin rate envelope between $\omega_{\min }$ and $\omega_{\max }$.

\section{Crowdsourcing}

\section{Model Validation and Refinement}

The digital twin is so far predicated on the rudimentary design $\Gamma$ in real space (Figure 2 ), and the critical spin rate $\Omega(5)$ and its standard deviation $\Delta \Omega(6)$ in frequency space (Figure 3). A community-driven approach organized by blockchain (Figure 1 ) needs to experimentally characterize this basic version of a CP-DF valve. Followingly, improvements to the modelling, e.g., to include more degrees of freedom in the layout, hydrodynamics, flexibility of the lid, capillary action, or enclosed gas pockets.

While the layout in Figure 2 is composed of cuboidal segments so the volume integral for $U$ (2) can be substituted by an algebraic formula in a quasi-hydrostatic approximation, these refinements will require a substantial upgrade of the computational method to full-fledged (numerical) solvers, e.g., as part of computational fluid dynamics (CFD) software packages. In addition, boundary conditions (Table 1) may need to be defined, e.g., for imposing limitations linked to the feasibility of materials, manufacture, assembly, fluidics, instrumentation, detection, or biochemistry.

For the crowdsourcing approach elaborated here, the proper delivery of these (significant) tasks is crypto-economically incentivized to attract honest quality contributions from researchers, validators and referee panels within the global crowd. Note that this foundational phase resembles the development of the blockchain ecosystem where a sufficiently large number of participants has to engage for making the community-based approach worthwhile, and workable. 


\section{Performance Optimization}

Once the model and simulation tools for the digital twin have been upgraded to properly virtualize the characteristics of the physical system, the design can be improved according to (a combination of) design goals that are expressed by quantitative key performance indicators (qKPIs).

Exemplary metrics reflecting these qKPIs are reducing the radial extension

$$
\bar{R}=\frac{R-\min \left[r_{0}(\omega)\right]}{R_{\max }-R_{\min }}
$$

for maximizing the number of sequential steps that can be integrated within the structurable area available between $R_{\min }$ and $R_{\max }$ (Table 1 ) on the disc, and the spatial footprint

$$
A_{\Gamma}=2 \pi \int_{\breve{r}}^{\hat{r}} w(r) d r
$$

of $\Gamma$ with its lateral extension $w(r)$ as a function of the radial position $r$; a small $A_{\Gamma}$ (8) allows increasing the spatial packing density, while a small band width $\Delta \Omega(6)$ implies potential for high-level multiplexing at good reliability. Also, the maximum field strength

$$
F_{\Omega}=m_{\text {part }} \cdot R_{\text {part }} \cdot \Omega^{2}
$$

that a particle, e.g., a red blood cell during plasma separation, of specific mass $m_{\text {part }}$ (with respect to its suspending medium) at a position $R_{\text {part }} \approx R$ experiences while rotating at the critical frequency $\omega=\Omega(5)$ of its downstream valve, might be of interest for reducing the time for assay completion. For a given particle mass $m_{\text {part }}$ determined with the biosample, and radial position $R$ that might be set available space in real and $\omega$-space, $F_{\Omega}$ (9) scales with the square of $\Omega \propto\langle\bar{r} \Delta r\rangle^{-1 / 2}(5)$, and, thus, configuring $\Gamma$ to minimize the radial product $\langle\bar{r} \Delta r\rangle(U, R, \Gamma, z=Z)$ in (1) for a given liquid of volume $U$ and density $\varrho$ would be commended.

With the significantly improved model emerging from the initial phase, the digital twin serves as a computational oracle to incentivize design improvements that can be objectively validated and ranked according to well-defined quantitative performance metrics, i.e., qKPIs (Figure 1). For awarding trust, the code representing the digital twin needs to be verifiable and openly accessible, e.g., from a common, cloud-based file storage system [70]; any member of the crowd has then the opportunity to execute the program, and be rewarded for providing outcomes to a smart contract. Similar to (delegated) proof-of-stake (PoS) [71] blockchains, the credibility of results ought to be assured by demanding crypto-economical "skin-in-the game", and requiring a minimum number of (independent) participants to this consensus finding mechanism.

In preceding publications, the model for CP-DF siphon valving has already been applied for design optimization of centrifugal microfluidic LoaD systems towards fluidic larger-scale integration [64], to enhance system-level reliability of multiplexed centrifugo-pneumatic flow control [65], derived anticounterfeit technologies [66], and on-board reagent storage and release by solvent-selective, rotationally opened membranes [67].

\section{Model Extension}

A recent review article broadened the scope of the digital twin to, in principle, most passive and active flow control on centrifugal LoaD platforms [68]. Yet, the underling modelling needs to be significantly refined, experimentally validated, and then employed for expediting and de-risking the optimization of fluidic layouts within a large multiparameter space confined by practical boundary conditions, such as minimum wall thickness, or constrained by spin frequencies $\omega \leq \omega_{\max }$ and acceleration ramps $\partial \omega / \partial t$ that can be realized by the instrument-based spindle motor. This way, the digital twin may also 
foster standardization [72], in a sense that methods for design and testing are shared between all stakeholders along the value chain.

The digital twin approach has so far focused on centrifugal flow control. It can also be applied to describe LUOs. For instance, the speed of sedimentation of cells in plasma separation can be modelled as a function of the centrifugal field [73]. Moreover, the speed of rotationally induced mixing may be derived from fits to empirical data to avoid complicated CFD simulation on chaotic advection [74].

Beyond aspects of flow control and sample preparation, development of full-fledged applications certainly requires the incorporation of further domains into the digital twin. From a functional side, these tasks primarily concern the modelling of biochemical processes as well as optical or electrochemical detection. Last but not least, the simulation of manufacturing and assembly processes for the LoaD device, such as precision milling, 3D printing or mold flow analysis, including their tolerances and practical limits, would greatly enhance virtual device development.

\section{Summary and Outlook}

\section{Summary}

This paper has presented a new, highly inclusive community approach to leverage the benefits of the rapidly emerging blockchain technology for efficiently leading crowdsourced projects in fundamental science \& RTD to credible, high-quality outcomes, and for seeding widespread adoption. An essential prerequisite for linking to the blockchain are computational oracles which feed real-word information into the smart contracts, i.e., blockchain-engraved code that triggers transaction of cryptoassets, e.g., for rewarding or slashing stakes of the contributing parties.

The perspective is that, with sophisticated modelling and massive computing power becoming increasingly available for lease, e.g., through cloud resources, digital twins will be able to well represent the behavior of physical systems and processes; likewise, the crowd can validate parameters for their compliance to pre-defined quantitative key performance parameters (qKPIs) as expressed by a group of metrics. After the proper experimental validation of underlying models in the relevant physical domains, the digital twin acts as a virtual tool to verify reliability and performance goals within the code and methods documented in a foundational whitebook. To bestow mission-critical trust, verification is carried out by simulating the shared code underlying the digital twin by computational oracles constituted by a distributed network of independent, numerous, and staked computer.

On the analogy of crypto-economically incentivized decision making free of intermediates and hierarchies that are already well-established for running blockchains that have locked up more trillionscale USD-values without being directed by a distinguished individual, corporate or institutional leader, governance, arbitration and management of RTD projects can be efficiently orchestrated the global crowd.

\section{Outlook}

After optimizing the multi-domain modelling and simulation tools as recorded in a "whitebook" that is accessible to participants, and adapting proven governance structures, the project needs to engage a critical mass of stakeholders that covers a broad spectrum of roles withing the project. According to Metcalfe's law [75], such purposely inclusive spirit is conducive to stimulate exponential networking effects; it has also been proven that community-driven projects may are able to raise significant crowdfunding, even up to billions of US\$; these initiatives, whether aiming for financial goals or commons, might even be entirely orchestrated by code in so-called Decentralized Autonomous 
Organizations ("DAOs"), which have substantially improved over recent years, after having learnt some bitter lessons from early setbacks [26-32].

Such momentum, which has already been deemed unstoppable for blockchains like Bitcoin and Ethereum in the DeFi space, may decisively overcompensate the very much expected, substantial resistance among the vast majority of very traditionally minded scientists and technology developers. As common during the early phase of adoption of disruptive technologies, such "TradSci" community will overwhelmingly tend to stick to legacy structure, rendering fast adoption very challenging.

Yet, it will need to be seen whether, on the analogy of the digital currencies and artwork, also a culture of decentralized science ("DeSci") will gain clout; while details are hard to predict details, it is envisioned that tokenized artefacts, which may represent digitized ideas, intellectual property experimental and simulation results, data mining, models, program code, possible packaged by digital twins, will coin the way of organizing science \& RTD. Various, still somewhat incoherent and disparate initiatives have already been looming on the horizon, e.g., [76-81].

\section{Appendix}

Table 1 Default dimensions and boundary conditions for experimental parameters of the valving structure $\Gamma$ (Figure 2). They may serve as a starting point for optimization. The (inner) wall of the isoradial channel of axial length $\mathcal{L}$ and radial height $h$ is located at $R$. The inner, isoradial and inbound siphon sections have depths $d_{0}=d=d$, which lead to cross sections $A_{0}, A$ and $\mathcal{A}$ with respect to the (axial) direction of flow. As a first set for reflecting manufacturing restrictions, tolerances in vertical and lateral dimensions, and minimum wall thickness as well as the confinement of the structurable area between $R_{\min }$ and $R_{\max }$ are imposed for directing design optimization. The spread of loaded and on-disc metered volumes $U$ is represented by $\Delta U$, the density $\varrho$ corresponds to water at room temperature, and $p_{0}$ to standard atmospheric pressure.

\begin{tabular}{|l|c|c|c|}
\hline Isoradial Channel & $R=30 \mathrm{~mm}$ & $\mathcal{L}=30 \mathrm{~mm}$ & $h=3 \mathrm{~mm}$ \\
\hline $\begin{array}{l}\text { Cross Sections } \\
\text { (depth } \times \text { width) }\end{array}$ & \multicolumn{2}{|c|}{$A_{0}=A=1 \mathrm{~mm} \times 10 \mathrm{~mm}$} & $\mathcal{A}=1 \mathrm{~mm} \times 1 \mathrm{~mm}$ \\
\hline Depths & \multicolumn{3}{|c|}{$d_{0}=d=d=1 \mathrm{~mm}$} \\
\hline Minimum Dimensions & Vertical $\geq 300 \mu \mathrm{m}$ & Lateral $\geq 200 \mu \mathrm{m}$ & Wall Thickness $\geq 1 \mathrm{~mm}$ \\
\hline Structurable area: & $R_{\min }=7.5 \mathrm{~mm}$ & $R_{\max }=55 \mathrm{~mm}$ & \\
\hline Geom. Tolerances & Vertical: $30 \mu \mathrm{m}$ & Lateral: $20 \mu \mathrm{m}$ & \\
\hline Liquid Volume & $U=100 \mu \mathrm{l}$ & $\Delta U=100 \mathrm{nl}$ & $\varrho=997 \mathrm{~kg} \cdot \mathrm{m}^{-3}$ \\
\hline Ambient pressure & \multicolumn{3}{|c|}{$p_{0}=p_{\text {std }}=1013.25 \mathrm{hPa}$} \\
\hline
\end{tabular}

\section{References}

1. Nakamoto, S. Bitcoin: A Peer-to-Peer Electronic Cash System. 2009 Published: 24/05/2009; Accessed: 15/09/2020; Available on: https://bitcoin.org/bitcoin.pdf.

2. Mellor, S. Despite El Salvador's bumpy Bitcoin rollout, a queue of countries forge ahead with legalizing crypto. 2021; Accessed: 29/09/2021; Available on: https://finance.yahoo.com/news/despite-el-salvador-bumpy-bitcoin-123822173.html.

3. Cuthbertson, A. Global bitcoin trend continues as Ukraine and Panama look to follow El Salvador's lead. 2021 Published: 09/09/2021; Accessed: 29/09/2021; Available on: https://www.independent.co.uk/life-style/gadgets-and-tech/bitcoin-ukraine-panamacrypto-b1917270.html.

4. Ossinger, J. and Z. Huang. Chinese regulators are serious about crypto ban this time. 2021; Accessed: 29/09/2021; Available on: https://www.bloomberg.com/news/articles/2021-0926/chinese-regulators-are-serious-about-banning-crypto-this-time.

5. Ahmed, A. and N. Anand. India to propose cryptocurrency ban, penalising miners, traders source. 2021 Published: 14/09/2021; Accessed: 29/09/2021; Available on: https://www.reuters.com/article/uk-india-cryptocurrency-ban-idUSKBN2B60QP. 
6. Suberg, W. China fear is now infrastructure bill fear -5 things to watch in Bitcoin this week. $2021 \quad$ Published: 27/09/2021; Accessed: 29/09/2021; Available on: https://cointelegraph.com/news/china-fear-is-now-infrastructure-bill-fear-5-things-towatch-in-bitcoin-this-week.

7. Smart contract. Accessed: 09/11/2020; Available on: https://en.wikipedia.org/wiki/Smart contract.

8. Szabo, N. Formalizing and Securing Relationships on Public Networks. First Monday, 1997. 2, DOI: 10.5210/fm.v2i9.548.

9. Buterin, V. White Paper ethereum/wiki Wiki - GitHub. 2014; Accessed: 24/07/2020; Available on: https://github.com/ethereum/wiki/wiki/White-Paper.

10. Cardano. Accessed: 27/09/2021; Available on: https://cardano.org/.

11. Polkadot. Accessed: 27/09/2021; Available on: https://polkadot.network/.

12. Solana. Accessed: 27/09/2021; Available on: https://solana.com/.

13. Tezos. Accessed: 30/09/2021; Available on: https://tezos.com/.

14. Avalanche. Accessed: 01/10/2021; Available on: https://www.avax.network/.

15. Algorand. Accessed: 01/10/2021; Available on: https://www.algorand.com/.

16. Proof-of-work. Accessed: 29/09/2021; Available on: https://en.bitcoinwiki.org/wiki/Proof-ofwork.

17. Proof-of-stake. Accessed: 29/09/2021; Available on: https://en.bitcoinwiki.org/wiki/Proof-ofstake.

18. Blockchain Oracles. Accessed: 24/07/2020; Available on: https://blockchainhub.net/blockchain-oracles/.

19. CHAINLINK - Your smart contracts connected to real world data, events and payments. 2020; Accessed: 24/07/2020; Available on: https://chain.link/.

20. Kapilkov, M. A new Cardano-based project is handling oracles a lot differently than Chainlink. Cointelegraph, 2020.

21. Blockchain oracles explained. 2021 Published: 29/04/2021; Accessed: 27/09/2021; Available on: https://academy.binance.com/en/articles/blockchain-oracles-explained.

22. Band protocol - Secure, Scalable Blockchain-Agnostic Decentralized Oracle. Accessed: 17/08/2020; Available on: https://bandprotocol.com/.

23. Helium - People-powered networks. Start a wireless revolution. Accessed: 24/08/2021; Available on: https://www.helium.com/.

24. IOTA Foundation - Redifining trust, value and ownership. Accessed: 27/09/2020; Available on: https://www.iota.org/.

25. Decentralized autonomous organization. Accessed: 14/08/2020; Available on: https://en.wikipedia.org/wiki/Decentralized autonomous organization.

26. Moloch DAO explained: Using self-interest to Ethereum's advantage. Accessed: 18/08/2020; Available on: https://concourseopen.com/blog/moloch-dao-explained/.

27. DAOstack is an open source project advancing the technology and adoption of decentralized governance.; Accessed: 17/08/2020; Available on: https://daostack.io/.

28. Dale, B. and W. Foxley Story from Tech DeFi Leader MakerDAO Weighs Emergency Shutdown Following ETH Price Drop. 2020.

29. Peng, T. A Community-Governed DeFi Platform Makes Crowdfunding Decentralized (Mantra DAO). Cointelegraph, 2020.

30. The Genesis DAO. Accessed: 18/08/2020; Available on: https://daostack.io/genesis.

31. Vigna, P. Chiefless Company Rakes In More Than $\$ 100$ Million - Group called DAO is running itself via computer code. The Wall Street Journal, 2016. 2020.

32. Siegel, D. Understanding The DAO Attack. coindesk, 2016. 2020.

33. Digital Twin. 2021; Accessed: 25/05/2021; Available on: https://en.wikipedia.org/wiki/Digital twin. 
34. Grieves, M. and J. Vickers, Digital Twin: Mitigating Unpredictable, Undesirable Emergent Behavior in Complex Systems, in Transdisciplinary Perspectives on Complex Systems: New Findings and Approaches, F.-J. Kahlen, S. Flumerfelt, and A. Alves, Editors. 2017, Springer International Publishing: Cham. p. 85-113.

35. Marr, B. What Is Digital Twin Technology - And Why Is It So Important? 2017 Published: 06/03/2017; Accessed: 25/05/2021; Available on: https://www.forbes.com/sites/bernardmarr/2017/03/06/what-is-digital-twin-technologyand-why-is-it-so-important/.

36. Bitcoin improvement proposals (BIPS). Accessed: 06/10/2021; Available on: https://github.com/bitcoin/bips.

37. Ethereum Improvement Proposals (EIPs) describe standards for the Ethereum platform, including core protocol specifications, client APIs, and contract standards.; Accessed: 06/10/2021; Available on: https://eips.ethereum.org/.

38. Augur - Put your skills to the test and WIN! 2020; Accessed: 24/07/2020; Available on: https://www.augur.net/.

39. Entriken, W., D. Shirley, J. Evans, and N. Sachs EIP-721: ERC-721 Non-Fungible Token Standard. Ethereum Improvement Proposals, 2020.

40. Non-fungible token. Accessed: 04/01/2021; Available on: https://en.wikipedia.org/wiki/Nonfungible token.

41. Kramer, M. What are non-fungible tokens? (NFTs). Available on: https://decrypt.co/resources/non-fungible-tokens-nfts-explained-guide-learn-blockchain.

42. Organizations of the future run on Aragon. Accessed: 24/07/2020; Available on: https://aragon.org/.

43. Introduction to Ethereum governance. Accessed: 30/09/2021; Available on: https://ethereum.org/en/governance/.

44. Cardano - Governance. Accessed: 30/09/2021; Available on: https://cardano.org/governance/.

45. Solana - Governance. Accessed: 30/09/2021; Available on: https://forums.solana.com/c/governance/10.

46. Polkadot - Governance. Accessed: 30/09/2021; Available on: https://wiki.polkadot.network/docs/learn-governance.

47. Hedera; Accessed: 05/10/2021; Available on: https://hedera.com/.

48. Ethereum is a global, open-source platform for decentralized applications. Accessed: 14/08/2020; Available on: https://ethereum.org/.

49. Cardano. Accessed: 03/09/2020; Available on: https://cardano.org/.

50. eosio. Available on: https://eos.io/.

51. Reputation system. Accessed: 28/08/2020; Available on: https://en.wikipedia.org/wiki/Reputation system.

52. Hirsch, J.E. An index to quantify an individual's scientific research output. Proc Natl Acad Sci U S A, 2005. 102, 16569-72 DOI: 10.1073/pnas.0507655102.

53. Powers, B. New MIT Paper Roundly Rejects Blockchain Voting as Solution to Election Woes. $2020 \quad$ Published: 20/11/2020; Accessed: 06/10/2021; Available on: https://www.coindesk.com/tech/2020/11/16/new-mit-paper-roundly-rejects-blockchainvoting-as-solution-to-election-woes/.

54. Buterin, V., Z. Hitzig, and E.G. Weyl Liberal Radicalism: Formal Rules for a Society Neutral Among Communities. SSRN Electronic Journal, 2018. DOI: 10.2139/ssrn.3243656.

55. Lalley, S. and E.G. Weyl, Quadratic Voting: How Mechanism Design Can Radicalize Democracy (December 24, 2017). American Economic Association Papers and Proceedings, Vol. 1, No. 1, 2018, Available at SSRN: https://ssrn.com/abstract=2003531 or http://dx.doi.org/10.2139/ssrn.2003531. Quadratic Voting: How Mechanism Design Can 
Radicalize Democracy (December 24, 2017). American Economic Association Papers and Proceedings, 2018. 1, DOI: 10.2139/ssrn.2003531.

56. Buterin, V. Quadratic Payments: A Primer. 2019 Published: 07/12/2019; Accessed: 06/10/2021; Available on: https://vitalik.ca/general/2019/12/07/quadratic.html.

57. Rouse, M. Trusted Platform Module (TPM). Accessed: July 24; Available on: https://whatis.techtarget.com/definition/trusted-platform-module-TPM.

58. Ducrée, J., M. Etzrodt, B. Gordijn, M. Gravitt, S. Bartling, R. Walshe, and T. Harrington Blockchain for Organising Effective Grass-Roots Actions on a Global Commons: Saving The Planet. Frontiers in Blockchain, 2020. 3, 33 DOI: 10.3389/fbloc.2020.00033.

59. Ducrée, J., M. Gravitt, R. Walshe, S. Bartling, M. Etzrodt, and T. Harrington Open Platform Concept for Blockchain-Enabled Crowdsourcing of Technology Development and Supply Chains. Frontiers in Blockchain, 2020. 3, 386525 DOI: 10.3389/fbloc.2020.586525.

60. Ducrée, J. Research - A blockchain of knowledge? Blockchain: Research and Applications, 2020. 1, 100005 DOI: 10.1016/j.bcra.2020.100005.

61. Ducrée, J., M. Etzrodt, S. Bartling, R. Walshe, T. Harrington, N. Wittek, S. Posth, K. Wittek, A. Ionita, W. Prinz, and J. Lawton Unchaining Collective Intelligence for Science, Research and Technology Development by Blockchain-Boosted Community Participation. Frontiers in Blockchain, 2020. 4, 631648 DOI: 10.3389/fbloc.2021.631648.

62. Ducrée, J., M. Etzrodt, S. Bartling, R. Walshe, T. Harrington, N. Wittek, S. Posth, K.W.A. Ionita, W. Prinz, D. Kogias, T. Paixão, I. Peterfi, and J. Lawton Unchaining collective intelligence for science, research and technology development by blockchain-boosted community participation. Frontiers in Blockchain, 2021. DOI: 10.3389/fbloc.2021.631648.

63. Kshirsagar, M., Y. Yao, G. Vaidya, J. Ducrée, and C. Ryan Convergence of blockchain, autonomous agents and knowledge graph to share electronic health records. Frontiers in Blockchain, 2021. DOI: 10.3389/fbloc.2021.661238.

64. Ducrée, J. Design optimization of centrifugal microfluidic "Lab-on-a-Disc" systems towards fluidic larger-scale integration. Appled Sciences, 2021. 11, 5839 DOI: 10.3390/app11135839.

65. Ducrée, J. Secure air traffic control at the hub of multiplexing on the centrifugo-pneumatic Labon-a-Disc platform. Micromachines, 2021. 12, 700 DOI: 10.3390/mi12060700.

66. Ducrée, J. Anti-counterfeit technologies for microfluidic "Lab-on-a-Disc" systems. Sensors \& Actuators A - Physical, 2021. DOI: 10.20944/preprints202107.0443.v1.

67. Ducrée, J. On-board reagent storage and release by solvent-selective, rotationally opened membranes - A digital twin approach. Microfluidics and Nanofluidics, 2021. DOI: https://doi.org/10.33774/chemrxiv-2021-pvb0t.

68. Ducrée, J. Systematic review of centrifugal valving based on digital twin modelling towards highly integrated Lab-on-a-Disc systems. Nature Microsystems \& Nanoengineering, 2021. DOI: 10.20944/preprints202105.0683.v2.

69. Ducrée, J. Efficient development of integrated Lab-On-A-Chip systems featuring operational robustness and nanufacturability. Micromachines, 2019. 10, 12 DOI: 10.3390/mi10120886.

70. Github. Accessed: 04/10/2021; Available on: https://github.com/.

71. Proof of Stake. Accessed: 09/11/2020; Available on: https://en.wikipedia.org/wiki/Proof of stake.

72. Reyes, D.R., H.v. Heeren, S. Guha, L.H. Herbertson, A.P. Tzannis, J. Ducrée, H. Bissig, and H. Becker Accelerating Innovation and Commercialization Through Standardization of Microfluidic-Based Medical Devices. Lab on a Chip, 2021. 21, 9-21 DOI: 10.1039/DOLC00963F.

73. Haeberle, S., T. Brenner, R. Zengerle, and J. Ducrée Centrifugal extraction of plasma from whole blood on a rotating disk. Lab on a Chip, 2006. 6, 776-781 DOI: 10.1039/b604145k.

74. Grumann, M., A. Geipel, L. Riegger, R. Zengerle, and J. Ducree Batch-mode mixing on centrifugal microfluidic platforms (vol 5, pg 560, 2005). Lab on a Chip, 2005. 5, 1184-1184 DOI: 10.1039/b418253g. 
75. Metcalfe's law. 2021; Accessed: 25/09/2021; Available on: https://en.wikipedia.org/wiki/Metcalfe\%27s law.

76. Sanders, R. UC Berkeley will auction NFTs of Nobel Prize-winning inventions to fund research. $2021 \quad$ Published: 27/05/2021; Accessed: 07/10/2021; Available on: https://news.berkeley.edu/2021/05/27/uc-berkeley-will-auction-nfts-of-nobel-prizewinning-inventions-to-fund-research/.

77. Bloxberg: Blockchain Infrastructure for Scientific Research. 2020; Accessed: 24/07/2020; Available on: https://bloxberg.org/.

78. Bartling, S. Blockchain for Science. Accessed: 24/07/2020; Available on: https://www.blockchainforscience.com/.

79. ARTIFACTS - Researcher recognition. Accelerated. Accessed: 24/07/2020; Available on: https://artifacts.ai/.

80. DEIP.world - Open Innovation Network. 2020; Accessed: 24/07/2020; Available on: https://deip.world/.

81. DeSci - Decentralized Science. 2021; Accessed: 07/10/2021; Available on: https://www.desci.nft. 